\title{
Selection on reproductive characters: floral morphology in Asclepias syriaca
}

\author{
M. T. MORGAN* \& D. J. SCHOEN \\ Department of Biology, McGill University, Montréal, Quebec, Canada H3A 1B1
}

\begin{abstract}
Pollinia insertions and removals represent a convenient index of male and female fertilities in the Asclepiadaceae. This fact, in conjunction with regression analysis, allows estimation and comparison of gender-specific selection acting on floral characters in Asclepias syriaca. Univariate regression analysis indicates statistically significant selection on minute aspects of floral morphology through the male and especially female reproductive pathways. Analyses using multiple regression to correct for overall plant size do not qualitatively change the univariate results. The relatively large number of statistically significant selection gradients suggests active adaptation of floral morphological features to the pollinator fauna. Selection through female function differs significantly from selection on the same trait through male function for four traits, emphasizing an antagonism between genders in hermaphrodites. This antagonism and the absence of selection of the antagonistic traits through net fertility are consistent with an equilibrium quantitative genetic perspective, while greater opportunity for selection through female function is counter to expectations based on Bateman's principle of sexual selection.
\end{abstract}

Keywords: Asclepias syriaca, hermaphrodite, reproduction, sexual selection.

\section{Introduction}

Floral characters of hermaphroditic flowering plants evolve in response to selection acting simultaneously through male and female sexual functions. This is in contrast to nonreproductive characters and organisms with separate sexes, where a single measure of selection is appropriate. Until recently, few studies have used a methodology incorporating this unique feature of hermaphrodite character evolution. Instead, studies have focused on only the female part of the fitness equation, or attempted to cast questions about the evolution of morphological characters into an abstract currency of 'allocation' (Morgan, 1994). Development of quantitative genetics theory (Lande, 1976, 1979) and its application to the evolution of reproductive characters in hermaphrodites (Morgan, 1992) provides a set of tools for appropriate analysis of floral morphological characters. The quantitative genetic formulation emphasizes the distinction between selection, appropriately measured under field conditions, and inheritance, usually determined through breeding programmes under environmentally controlled conditions (Arnold, 1983). Quantitative genetics identifies regression analysis as the appropriate tool for documenting selection (Lande \& Arnold, 1983). Applica-

\footnotetext{
*Current address and correspondence: Department of Botany/ Department of Genetics and Cell Biology, Washington State University, Pullman, WA 99164-4238, U.S.A. E-mail: mmorgan@mail.wsu.edu
}

tion of the quantitative genetic formulation to hermaphrodites emphasizes both the importance of gender-specific relative fertilities as the currency of evolution, and the equal contributions of selection through male and female functions to character change (Morgan, 1992).

Asclepias syriaca and other milkweeds (Asclepiadaceae) are uniquely suited to measurement of selection on floral characters. The elaborate floral morphology of $A$. syriaca provides a stage for an intricate pollination biology (Fig. 1; Robertson, 1886; Wyatt \& Broyles, 1990). Inflorescences consist of many flowers, each of which has a 'corona' made up of five cup-shaped 'hoods' and associated 'horns' extending from the base of the hood toward the centre of the flower (gynostegium). Pollen grains are packaged into pollinia, each pollinium consisting of two pollen sacks joined by translator arms and a corpusculum; only the corpusculum is exposed to pollinators. Hoods accumulate copious nectar (Willson \& Bertin, 1979; Wyatt et al., 1992), and the arrangement of the hoods around the gynostegium directs hairs of foraging pollinator limbs and other body parts toward a small groove in the corpusculum. The hairs become wedged in the groove, and the corpusculum and associated pollinia become dislodged as the pollinator leaves the flower (Robertson, 1886). During pollinator flight, the translator arms dehydrate and the orientation of the pollinia changes so that when pollinators visit 


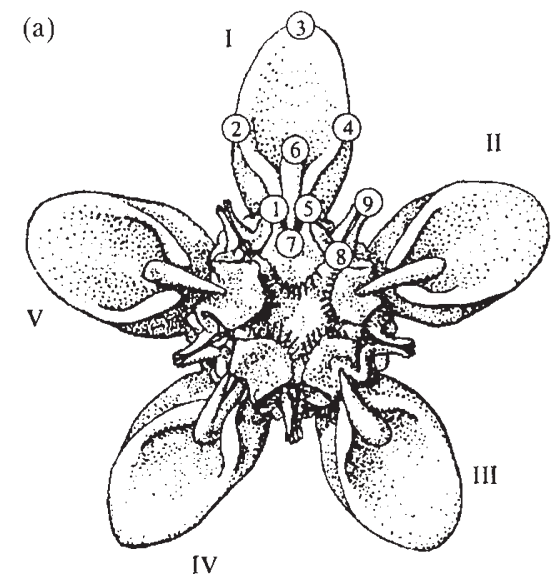

(b)

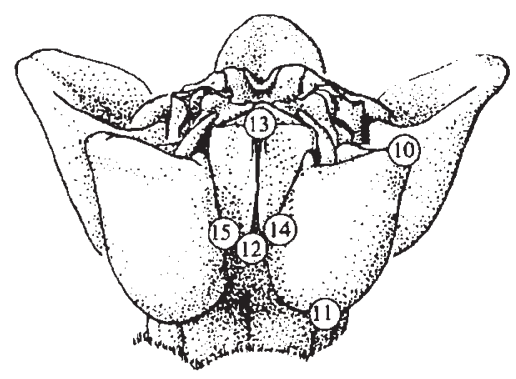

another inflorescence the pollinium may insert into the stigmatic slit and juxtapose against the stigmatic surface. Previous studies of $A$. syriaca and other milkweeds focus on the consequences of inflorescence size for fertility (Willson \& Rathcke, 1974; Willson \& Price, 1977; Broyles \& Wyatt, 1990, 1995), despite the unique opportunity to address morphological evolution.

Two contrasting interpretations of selection on reproductive characters involve Bateman's principle (Bateman, 1948) and equilibrium quantitative genetic or phenotypic formulations (Lloyd, 1985; Morgan, 1992, 1994). Bateman suggests that resources often limit female reproductive success, whereas mating opportunities determine male fertility. Consistent with Bateman's characterization of resource-limited female function, in Asclepias low levels of fruit-set (Willson \& Rathcke, 1974; Willson \& Price, 1977; Queller, 1983, 1985; Bookman, 1984,) are easily enhanced by resource supplementation (Willson \& Price, 1977; Queller, 1985). Increasing inflorescence size enhances pollinia donation (Broyles \& Wyatt, 1990, 1995), as envisaged by the second portion of Bateman's scenario. As a consequence, reproductive characters of $A$. syriaca might benefit pollen donation more than pollen receipt. Analysis of a quantitative genetic model (or determination of evolutionary stable strategies in phenotypic models), on the other hand, shows that characters influencing male and female reproductive success evolve so that the selection gradient through one gender is equal in magnitude but opposite in sign to the selection gradient through the other gender (Morgan, 1992). Under this equilibrium perspective, inflorescence or floral reproductive characters have equal consequence for male and female fertilities separately, but have no consequence for net (male plus female) fertility.
Fig. 1 Floral morphology and recorded landmarks in Asclepias syriaca. Flowers consist of five units (numbered I to V), each with a 'hood' (outlined by points 1, 2, 3, 4 and 5) and a 'horn' (inserted into the hood at 6 , extending toward the centre of the flower to 7). The corpusculum is located at 8 ; pollinia insert into the stigmatic slit 9 . In the lateral view points 10,11 and 14 partially outline the hood; the stigmatic slit extends from 12 to 13 . Adapted from Wood (1974).
The aim of this study is to compare selection acting on floral reproductive characters in $A$. syriaca through male and female functions. We use pollinia insertions and removals as indices of fertility, and regression analysis to document selection on a suite of reproductive characters. Statistical comparisons of selection gradients estimated from the regression analysis allow us to evaluate the relative roles of Bateman's principle and the equilibrium quantitative genetic perspective. Results of the investigation illustrate the utility of the regression approach in documenting selection, and in evaluating differing hypotheses for the evolution of elaborate floral morphology in the Asclepiadaceae.

\section{Materials and Methods}

\section{Species and study site}

Asclepias syriaca is a self-incompatible (Morse, 1994) perennial milkweed found throughout north-eastern North America. Plants are 1-2 m high, producing a single stem with 1-5 axial inflorescences of 30-100 flowers; each intricately constructed 5 -merose flower has a diameter of $10-15 \mathrm{~mm}$. Inflorescences on an individual plant mature sequentially, flowers in each inflorescence reach anthesis over the course of 2 days, and individual flowers persist for 4-5 days (Morse, 1994; personal observation).

The study population is located in an abandoned field surrounded by second growth forest on Mont St. Hilaire, a United Nations biosphere preserve located in the Monteregian Hills south of Montréal, Canada. The field site is occasionally managed and is open to the public, although few visitors are present during the flowering time of $A$. syriaca. Several thousand ramets of $A$. syriaca occur at varying densities over an area of approximately $500 \times 200 \mathrm{~m}$. A number of clones occur in the study 
population, as evidenced by the dectection of individuals with different multilocus allozyme genotypes in a preliminary electrophoretic survey (M. T. Morgan \& D. J. Schoen, unpublished information).

\section{Sampling and morphological measures}

Reproductive characters and fertilities were measured on 500 different ramets of $A$. syriaca. Plants were chosen arbitrarily from throughout the field site on 8 July (200 plants) and 11 July ( 300 plants). On both days, inflorescences with 75-90 per cent of flowers open were chosen. Preliminary observation indicates that anthesis in these flowers started within the previous 2 days. The number of mature inflorescences and the order of initiation of the focal inflorescence (e.g. second inflorescence on the plant to start flowering) were recorded, and a single flower from each inflorescence was removed. The corona was scored visually for intensity of 'pigment', ranging from 0 (white) to 3 (deep pink), and for presence of a 'target', in which the gynostegium colour contrasts with the colour of the corona ( 1 indicates target presence, 0 target absence). The flower was then placed in an Eppendorf tube with $1 \mathrm{~mL} 1$ per cent $\mathrm{HCl}$ in methanol for later densitometer analysis. Five days after marking them in the field, inflorescences were collected and preserved ('pickled') in jars with ethanol acetic acid sponges. Pollinator activity during this time led to many but not all pollinia being removed.

Flowers collected in Eppendorf tubes were used for quantitative assessment of floral pigmentation. Fifteen days after collection, the flowers were sorted into seven 'visual ranks' based on colour of the $\mathrm{HCl}$ methanol solution (a score of 1 corresponding to light and 7 to dark). Pigmentation was further quantified by densitometer assessment of optical density at a wavelength of $525 \mathrm{~nm}$. There is a peak in the optical density at this wavelength.

Morphological characters were measured on individual flowers chosen arbitrarily from the pickled inflorescences. The number of flowers on the inflorescence was determined by counting pedicels. Lateral and top views of a single flower from each inflorescence were obtained using a dissecting microscope and computer imaging software. Points indicated on Fig. 1 were digitized, and a series of measurements derived (Table 1). The measurements involve overall flower size ('area'), hood characters (average length, width, height, separation between adjacent hoods and horn length), lateral height, length of the stigmatic slit, and length of the corpusculum. The five measurements of hood characters available from top views of each flower were reduced to a single average measurement.

\section{Fertility measures}

Female and male fertility measures were obtained by counting pollinia insertions and removals on five flowers chosen arbitrarily from the pickled inflorescences from which floral morphological measurements were taken. The extent of pollinia removal is easy to score because each flower produces exactly ten pollen sacs associated with five obvious corpuscula. Thus counting the number of remaining corpuscula gives an accurate estimate of pollinia

Table 1 Relation between floral landmarks and morphometric measurements in Asclepias syriaca. All linear measures of hood characters are averaged over hoods I-V

\begin{tabular}{ll}
\hline Character & Derivation from Fig. 1 \\
\hline $\begin{array}{l}\text { Floral size character } \\
\text { Flower area }\end{array}$ & Area enclosed by points I-8, II-8, .., V-8 \\
$\begin{array}{l}\text { Hood characters } \\
\text { Length }\end{array}$ & Distance from 3 to midpoint of 1 and 5 \\
Width & Distance from 2 to 4 \\
Height & Distance from 10 to 11 \\
Separation & Distance from I-4 to II-2, II-4 to III-2, etc. \\
Horn length & Distance from 6 to 7 \\
Lateral characters & \\
Stigmatic slit & Distance from 12 to 13 \\
Width & Distance from 14 to 15 \\
Corpusculum & \\
Length & Not shown \\
\hline
\end{tabular}


removal. Pollen germination occurs when a pollinium is inserted in a precise orientation into the stigmatic chamber (Galil \& Zeroni, 1969), but our assessment of female fertility is less stringent and involves insertion of pollinia into the stigmatic chamber regardless of orientation. Insertions were scored when a translator arm and attached corpusculum protrude from the stigmatic slit, or when the arm of a forceps passed through the stigmatic chamber dislodges a pollinium. Female fertility per flower ranges from 0 to 5 , because flowers have five stigmatic slits. Measurements of male and female fertilities on multiple flowers were combined into average male and female fertility estimates for the inflorescence, with numerical range between 0 and 5. The appropriateness of insertions and removals as indications of fertility receives attention in the Discussion.

\section{Statistical analysis}

A first goal of the statistical analysis is to document the relationship between measured floral characters and fertility. This is accomplished through simple linear regression of relative fertility on each character. Relative fertilities are simply the fertility of an individual divided by the average fertility of all individuals in the sample. Using relative fertilities makes the slopes of the estimated regression equations equivalent to selection gradients, $\hat{\beta}$, ofquantitative genetics (Lande \& Arnold, 1983). There are two types of fertility (male and female) and consequently two relative fertilities, with the relative fertility measures of the $i$ th individual being $W_{\mathrm{F}}(i) / \bar{W}_{\mathrm{F}}$ and $W_{\mathrm{M}}(i) / \bar{W}_{\mathrm{M}}$. The subscripts $\mathrm{F}$ and $\mathrm{M}$ refer to female and male. Number of pollinia insertions measures absolute female fertility $W_{\mathrm{F}}$, and number of removals indexes absolute male fertility $W_{\mathrm{M}}$.

Elasticities of selection (Morgan, unpubl.) are calculated to facilitate comparison of selection gradients acting on different traits. Selection gradients have a dimensionality equal to the inverse of the dimension of the measured trait (e.g. length measure in $\mathrm{mm}$ has selection gradient in units $\mathrm{mm}^{-1}$ ) so that dimensionality considerations alone often invalidate comparison of selection gradients. An elasticity of selection is a dimensionless number measuring the proportional change in fitness brought about by proportional change in character value; for instance, an elasticity of -0.1 means that a 10 per cent change in character value leads to a $-0.1 \times 10$ per cent $=-1$ per cent change in relative fitness. The minus sign indicates an inverse relation between character value and fitness, so that increas- ing the character value decreases fitness. Elasticities are mean-corrected measures of selection, just as the coefficient of variation is a mean-corrected measure of variation. Mathematically, the elasticity of trait $z$ with selection gradient $\beta$ and standard deviation $\sigma_{\hat{\beta}}$ is defined as $\hat{\beta}^{e}=\bar{z} \hat{\beta}$ with standard deviation $\bar{z} \sigma_{\beta}$. Statistical estimation of the elasticity is from regression of relative fertility on relative trait value (i.e. trait value of the individual divided by the average trait value of all individuals in the population). It is reasonable to compare elasticities of disparate characters to ask whether, for instance, a 10 per cent increase in optical density has as large an effect on fertility as a 10 per cent increase in horn length. Elasticities of a trait are compared by regressing the difference between relative male and female fertility on relative trait value, following the method for comparison of selection gradients outlined above.

Overall plant size is often an important component of fertility variation (e.g. individuals of $A$. exaltata with more flowers produce and set more seeds; Broyles \& Wyatt, 1990), but overall size may obscure interpretation of selection on other 'focal' characters. A series of multiple regressions was performed to correct for overall size and to address the relationship between focal characters and fertility. Each multiple regression includes as independent variables the size characters of Table 2 (number of inflorescences, number of pedicels and flower area) and a single additional focal character from the remainder of the table. The partial regression of the focal character with fertility reflects selection acting on the character independent of the size characters included in the analysis. The presentation of results focuses on the selection elasticities of the focal characters rather than presentation of the complete multiple regressions, because the goal is to identify selection acting on focal characters rather than to document the importance of selection on overall size.

Positive correlations between measured characters may result in inappropriately attributing statistical significance to several characters. As a partial solution to this we perform multiple regression including all measured characters, and report the partial regression coefficients showing significant linear association with relative fertilities. Multiple regression is not completely satisfactory, because significant partial regressions may be attributable to unmeasured characters (Lande \& Arnold, 1983, p. 1214).

Regression of the difference and the sum of relative fertilities on trait value aids in distinguishing 
between hypotheses for selection of reproductive characters. The interaction between trait value and fertility can be tested by subtracting female relative fertility from male relative fertility for each individual, and regressing this difference on relative trait value. Significant regressions indicate that the trait has a stronger effect on one gender than on the other (i.e. that the estimated selection elasticity through male function is significantly different from the selection elasticity through female function). Regressing the sum of male and female relative fertility on trait value evaluates whether there is net selection on total fitness. Statistical significance of this regression analysis is consistent with Bateman's principle, but contradicts the equilibrium expectation.

\section{Results}

Table 2 presents statistical summaries of the fertilities and characters measured. Plants in this population of $A$. syriaca produce about three inflorescences, with approximately 45 flowers per inflorescence. Size and visual characters show considerable variation, with coefficients of variation generally between 30 and 50 per cent. The large coefficient of variation associated with the 'target' occurs because this character has a binomial distribution with mean at the point maximizing variance. Linear measurements of flowers show the least variability, with coefficients of variation usually between 10 and 20 per cent. Individual flowers had slightly more than two pollinia removed and only one inserted. This implies that only half of the removed pollinia are successfully transferred between plants. Although the variance in estimated male fertility is larger than the variance in female fertility, the coefficient of variation shows the reverse pattern. There is thus greater opportunity for selection through female fertility than through male fertility (Crow, 1958; Arnold \& Wade, 1984).

Seven characters show statistically significant univariate selection gradients (Table 3 ). Six of the significant selection gradients are associated with female fertility (pollinia insertion) and two with male fertility (pollinia removal). Selection acts through both visual characters (pigment through female function; optical density through male function) and morphological characters (horn length, hood length, hood width, slit length and lateral

Table 2 Statistical characterization of fertilities and reproductive characters in Asclepias syriaca

\begin{tabular}{lcccc}
\hline Character & $\begin{array}{c}\text { Number of } \\
\text { individuals }\end{array}$ & $\begin{array}{c}\text { Mean } \\
\bar{x}\end{array}$ & $\begin{array}{c}\text { Variance } \\
\sigma^{2}\end{array}$ & $\begin{array}{c}\mathrm{CV} \\
100 \times \sigma / \bar{x}\end{array}$ \\
\cline { 5 - 6 } Fertilities & & & & \\
$\quad$ Female (insertions) & 444 & 1.15 & 0.41 & 56 \\
$\quad$ Male (removals) & 444 & 2.19 & 0.57 & 35 \\
Size characters & & & & \\
$\quad$ Number of inflorescences & 444 & 2.91 & 1.55 & 43 \\
$\quad$ Number of pedicels & 444 & 44.4 & 360.6 & 43 \\
$\quad$ Flower area (mm ${ }^{2}$ ) & 444 & 45.4 & 183.9 & 30 \\
Visual characters & & & & \\
Pigment & 444 & 2.01 & 0.058 & 12 \\
$\quad$ Target & 444 & 0.45 & 0.248 & 112 \\
$\quad$ Visual rank & 433 & 2.33 & 1.212 & 47 \\
$\quad$ Optical density & 435 & 0.14 & 0.003 & 37 \\
Hood characters & & & & \\
$\quad$ Length (mm) & 444 & 1.85 & 0.107 & 18 \\
$\quad$ Width (mm) & 444 & 1.32 & 0.021 & 11 \\
$\quad$ Height (mm) & 444 & 3.87 & 0.123 & 9 \\
$\quad$ Separation (mm) & 444 & 0.88 & 0.043 & 24 \\
$\quad$ Horn length (mm) & 444 & 0.93 & 0.053 & 25 \\
Insertion and corpusculum characters & & & & \\
$\quad$ Slit length (mm) & 444 & 2.31 & 0.019 & 6 \\
$\quad$ Width (mm) & 444 & 1.94 & 0.044 & 11 \\
$\quad$ Corpusculum length (mm) & 417 & 0.41 & 0.003 & 14 \\
\hline
\end{tabular}


Table 3 Univariate selection gradients $\hat{\beta}$, selection elasticities $\hat{\beta}^{c}$, and size-corrected elasticities $\tilde{\beta}^{c}$ through female and male sexual functions in Asclepias syriaca. Significance levels for size-corrected elasticities also apply to the corresponding elasticity and selection gradient

\begin{tabular}{|c|c|c|c|c|c|c|}
\hline Character & $\hat{\beta}_{\mathrm{F}}$ & $\hat{\beta}_{\mathrm{F}}^{e}$ & $\tilde{\beta}_{\mathrm{F}}^{c}$ & $\hat{\beta}_{\mathrm{M}}$ & $\hat{\beta}_{M}^{e}$ & $\tilde{\beta}_{\mathrm{M}}^{e}$ \\
\hline \multicolumn{7}{|l|}{ Size characters } \\
\hline Number of infiorescences & -0.0038 & -0.011 & & 0.0155 & 0.045 & \\
\hline Number of pedicels & 0.0008 & 0.036 & & -0.0016 & -0.072 & \\
\hline Flower area & 0.0007 & 0.034 & & -0.0006 & -0.029 & \\
\hline \multicolumn{7}{|l|}{ Visual characters } \\
\hline Pigment & -0.2443 & -0.491 & $-0.494^{*}$ & 0.0807 & 0.162 & 0.139 \\
\hline Target & 0.0872 & 0.039 & 0.038 & -0.0092 & -0.004 & 0.003 \\
\hline Visual rank & -0.0029 & -0.007 & -0.009 & -0.0085 & -0.020 & -0.017 \\
\hline Optical density & -0.0851 & -0.012 & -0.010 & 0.7855 & 0.110 & $0.086^{*}$ \\
\hline \multicolumn{7}{|l|}{ Hood characters } \\
\hline Length & 0.1937 & 0.358 & $0.363^{*}$ & 0.1323 & 0.244 & $0.209 *$ \\
\hline Width & 0.6300 & 0.834 & $0.836^{* * *}$ & 0.0801 & 0.106 & 0.169 \\
\hline Height & -0.0131 & -0.051 & -0.050 & 0.0333 & 0.129 & 0.100 \\
\hline Separation & 0.2171 & 0.190 & 0.206 & 0.0493 & 0.043 & 0.050 \\
\hline Horn length & 0.3302 & 0.306 & $0.320^{* *}$ & 0.0958 & 0.089 & 0.069 \\
\hline \multicolumn{7}{|c|}{ Insertion and corpusculum characters } \\
\hline Slit length & 0.4640 & 1.072 & $1.012 *$ & -0.1298 & -0.300 & -0.313 \\
\hline Width & 0.2585 & 0.500 & $0.504 *$ & 0.1233 & 0.239 & 0.202 \\
\hline Corpusculum length & 0.0533 & 0.022 & 0.006 & 0.2105 & 0.086 & 0.058 \\
\hline
\end{tabular}

${ }^{*} P<0.05 ; * * P<0.01 ;{ }^{* * *} P<0.001$.

width through female function; hood length through male function). None of the 'size' characters in Table 3 exhibits significant selection gradients.

Evolutionary elasticities range from -0.5 to about 1 (Table 3). The statistical transformation of selection gradients into elasticities is such that a gradient significantly different from zero means that the elasticity also differs from zero (although results from comparing one gradient with another are not necessarily informative about comparisons of elasticities). Therefore, statistical significances of the elasticities reported in Table 3 are the same as those of the corresponding selection gradients. Slit length and hood width show the largest elasticities, with relative changes in each of these characters leading to almost equivalent $(1.0,0.83$, respectively) relative increases in female fertility. Elasticities of selection through hood length and horn length are similar, whereas the selection gradients for these characters differ by a factor of 2 . The selection elasticity associated with pigment and female fertility is -0.49 , indicating that a 10 per cent increase in pigment leads to an approximately 5 per cent decrease in female fertility.

Multiple regressions including overall size $\left(\tilde{\beta}^{e}\right)$ do not alter the level of statistical significance of any selection elasticity. With the exception of the target character through male function, the signs of the size-corrected elasticities are the same as the univariate elasticities. The male elasticity associated with target changes from negative in the univariate analyses to positive in the size-corrected analyses, but in both cases the elasticity is far from statistical significance.

Including all characters in a multiple regression analysis changes the direction, magnitude and statistical significance of selection elasticities (Table 4). In the multiple regression analysis only three elasticities associated with female fertility are statistically significant, and none of the traits exhibits significant association with male fertility. Two of the significant multivariate selection elasticities (slit length and hood width) are comparable in sign and magnitude to their univariate counterparts. Amongst the visual characters the selection elasticity through target is significant in the multivariate but not univariate analysis; the pigment selection elasticity follows the reverse pattern.

Table 5 compares the action of selection through male and female functions for those characters with statistically significant size-corrected selection elasticities. Univariate analyses of size-corrected elasticities for four traits (pigment, hood width, horn length and slit length) show statistically significant inter- 
actions between male and female functions. Two of these traits (hood width and length) also exhibit a significant relation with net fitness, $\tilde{W}_{\mathrm{M}}+\tilde{W}_{\mathrm{F}}$. Multivariate regression shows statistically significant interaction effects for pigment, hood width and slit length elasticities, whereas coefficients associated with net fertility approach but do not reach significance for pigment and hood width.

Table 4 Multiple regression analysis of selection elasticities through female $\hat{\beta}_{\mathrm{F}}^{e}$ and male $\hat{\beta}_{\mathrm{M}}^{e}$ genders in Asclepias syriaca

\begin{tabular}{lrr}
\hline Character & $\hat{\beta}_{\mathrm{F}}^{e}$ & \multicolumn{1}{c}{$\hat{\beta}_{\mathrm{M}}^{e}$} \\
\hline Size characters & & \\
$\quad$ Number of inflorescences & -0.007 & 0.015 \\
Number of pedicels & 0.001 & -0.002 \\
Flower area & -1.594 & -0.023 \\
Visual characters & & \\
Pigment & -0.409 & 0.083 \\
Target & $0.054^{*}$ & 0.010 \\
Visual rank & -0.004 & -0.028 \\
Optical density & 0.063 & 0.102 \\
Hood characters & & \\
Length & 0.134 & 0.239 \\
Width & $0.678^{*}$ & 0.057 \\
Height & -0.247 & -0.047 \\
Separation & 0.268 & -0.016 \\
Horn length & 0.140 & -0.080 \\
Insertion and & & \\
corpusculum characters & & \\
Slit length & $1.023^{*}$ & -0.284 \\
Width & 0.025 & 0.041 \\
Corpusculum length & 0.041 & 0.138 \\
\hline
\end{tabular}

${ }^{*} P<0.05$.

\section{Discussion}

Selection operates on remarkably fine features of floral morphology in $A$. syriaca. Both pollinia insertions and removals have large fertility coefficients of variation (mathematically equivalent to the opportunity for selection; Crow, 1958; Arnold \& Wade, 1984). Six characters of individual flowers, especially those related to hood morphology, exhibit significant selection elasticities through female fertility (pollinia insertions). Male selection elasticities (pollinia removal) are statistically signficant for two additional hood characters. Analyses correcting for the influence of overall plant size further support the conclusion that the observational study undertaken here detects selective forces shaping floral morphology.

Regression analyses clarify the roles of Bateman's principle and the equilibrium perspective in the evolution of $A$. syriaca reproductive characters. Regressions of relative fertility on trait value indicate that selection acts primarily through female function, rather than through male function as suggested by Bateman's principle (Tables 3 and 4). In addition, the fertility coefficient of variation (i.e. the opportunity for selection) for pollinia insertion is larger than for pollinia removal. These observations suggest that current evolution of $A$. syriaca reproductive characters is occurring by selection through female rather than male function.

Comparisons of selection elasticities emphasize the antagonism between male and female functions in hermaphrodites. The effects of four of the eight size-corrected traits exhibiting significant univariate elasticities differ significantly between male and female fertilities in the univariate analyses (Table 5), and three of these remain significant in the multivariate analysis. Such antagonistic interactions are

Table 5 Tests for fertility interaction $\left(\tilde{W}_{\mathrm{M}}-\tilde{W}_{\mathrm{F}}\right)$ and for net fertility $\left(\tilde{W}_{\mathrm{M}}+\tilde{W}_{\mathrm{F}}\right)$ associated with size-corrected relative trait values in Asclepias syriaca

\begin{tabular}{lccccc}
\hline & \multicolumn{2}{c}{ Univariate } & & \multicolumn{2}{c}{ Multivariate } \\
\cline { 2 - 3 } Character & $\begin{array}{c}\text { Fertility } \\
\text { interaction }\end{array}$ & $\begin{array}{c}\text { Net } \\
\text { fertility }\end{array}$ & & $\begin{array}{c}\text { Fertility } \\
\text { interaction }\end{array}$ & $\begin{array}{c}\text { Net } \\
\text { fertility }\end{array}$ \\
\hline Pigment & $0.463^{* *}$ & -0.174 & & $0.339^{*}$ & $-0.261^{+}$ \\
Optical density & 0.106 & 0.046 & & 0.004 & 0.103 \\
Hood length & -0.148 & $0.300^{* *}$ & & 0.117 & 0.167 \\
Hood width & $-0.727^{* *}$ & $0.476^{* *}$ & & $-0.715^{*}$ & $0.395^{+}$ \\
Horn length & $-0.276^{* *}$ & $0.202^{*}$ & & -0.222 & 0.025 \\
Slit length & $-1.284^{* *}$ & 0.410 & & $-1.284^{* *}$ & 0.363 \\
Insertion width & -0.278 & $0.371^{*}$ & & 0.039 & 0.030 \\
\hline
\end{tabular}

${ }^{+} P<0.1 ;{ }^{*} P<0.05 ;{ }^{*} P<0.01$. 
consistent with both Bateman's and the equilibrium perspective. Of the four characters showing interaction in the univariate analysis, though, only two exhibit significant elasticities associated with net fertility. No characters show significant net elasticities in the multivariate analyses (Table 5). These patterns of selection are consistent with the equilibrium hypothesis, which anticipates no net selection on reproductive characters (Morgan, 1992). However, the observation of significant net selection for two antagonistic characters in the univariate analyses, and inability to identify relatively large elasticities as statistically significant in the multivariate analysis, undermine to some extent the strength of this conclusion.

\section{Pollinia and fertility}

Pollinia insertions and removals are used to index fertility, but does the complex pollination biology of $A$. syriaca undermine this assay? Empirical observations suggest not. From the perspective of female fertility, incrementing the number of pollinia insertions through hand pollination increases the probability of fruit-set by an order of magnitude in some milkweeds (Wyatt, 1976, 1981; Queller, 1985), and doubles fruit-set in $A$. syriaca (Morse \& Fritz, 1983). Empirical evidence also indicates that pollinia removal is a reasonable index of male fertility, because removal strongly correlates with number of seeds set in A. exaltata (Broyles \& Wyatt, 1990) and in the orchid Aerangis ellisii pollinia removal strongly correlates with donation success (recovery of microtagged pollinia on stigmas of other plants; Nilsson et al., 1992). Empirical evidence offers strong support for the utility of pollinia insertion and removal as indicators of female and male fertility.

Our results are likely to be robust to many of the complexities of the reproductive biology of $A$. syriaca. Removed pollinia often do not get inserted into stigmatic chambers (Table 2; Piper \& Waite, 1988; Nilsson et al., 1992) and a prodigiously small fraction of flowers mature fruit following pollinia insertion (Willson \& Bertin, 1979; Bookman, 1984). However, relative fertilities are the currency of evolution and are not affected by such absolute measures of fertility; for instance, suppose that only 10 per cent of removed pollinia reinsert so that the absolute male fertility of all individuals is only 10 per cent of that measured by pollinia removals, the male fertility of the $i$ th individual is $0.1 W_{\mathrm{m}}(i)$, the average male fertility in the population is $0.1 \bar{W}_{\mathrm{M}}$, and the relative fertility remains unchanged at $W_{\mathrm{M}}(i) / \bar{W}_{\mathrm{M}}$. Snow (1989) comments on this aspect of a previous study (Piper \& Waite, 1988). The potential for clonal geitonogamy (Wyatt, 1981; Pleasants, 1991; Shore, 1993) in conjunction with self-incompatibility (Wyatt, 1976) and the restricted number of sites for pollinia insertion available per flower (Wyatt, 1978; Broyles \& Wyatt, 1990; Kahn \& Morse, 1991), mean that there is likely to be a nonlinear relationship between the number of pollinia insertions and female fertility. Analysis using selection gradients represents a first-order approximation of this complexity, and is unlikely to mislead when the nonlinear effects are as subtle as those suggested for $A$. syriaca. This is especially true when environmental variation presents noise that obscures complex relationships between trait values, indices of fertility and actual fitness.

\section{Evolution of reproductive characters}

The relatively large number of statistically significant selection elasticities suggests active adaptation of floral morphological features to the pollinator fauna. Why do characters so intimately related to fitness appear to differ from their optimum value? Pervasive directional selection might arise if the plants included in the present study experienced an atypical pollinator fauna. An atypical fauna might represent a short-term aberration, so that suites of floral characters with high fertility in one breeding season might not be selectively advantageous in another. A multi-year study would find different patterns of selection in each year, and the net effect might reduce strong or pervasive selection observed in any one year to more moderate or stabilizing selection over evolutionarily relevant time periods. Asclepias syriaca is introduced to North America and the pollinator fauna might be atypical because of its difference from the fauna in Eurasia. In this case a multi-year study would find similar patterns of selection each year; documenting selection in milkweeds native to North America would provide an interesting contrast to the results found in $A$. syriaca. Pervasive selection might persist over evolutionary time if heritable genetic variation for selected characters is absent. This seems unlikely because of the almost universal finding of significant trait heritabilities (Falconer, 1981), even for minute features of morphology (Weber, 1992). Nonetheless, the reproductive biology of $A$. syriaca ensures that the hundreds of seeds within each fruit are full-sibs (Broyles \& Wyatt, 1990), allowing estimation of quantitative genetic parameters from naturally pollinated progeny arrays.

(C) The Genetical Society of Great Britain, Heredity, 79, 433-441. 


\section{Acknowledgements}

Comments of an anonymous reviewer suggested the analysis in Table 5. We acknowledge the support of a National Sciences and Engineering Research Council of Canada postdoctoral fellowship (to M.T.M.) and operating grant (to D.J.S.), and FCAR (Quebec) team grant.

\section{References}

ARNOLD, s. J. 1983. Morphology, performance, and fitness. Am. Zool., 23, 347-361.

ARNOLD, s. J. AND WADE, M. J. 1984. On the measurement of natural and sexual selection: theory. Evolution, 38, 709-719.

BATEMAN, A. J. 1948. Intra-sexual selection in Drosophila. Heredity, 2, 349-368.

BOOKMAN, s. s. 1984. Evidence for selective fruit production in Asclepias. Evolution, 38, 72-86.

BROYLES, S. B. AND WYATT, R. 1990. Paternity analysis in a natural population of Asclepias exaltata: multiple paternity, functional gender, and the 'pollen-donation' hypothesis. Evolution, 44, 1454-1468.

BROYLES, S. B. AND WYATT, R. 1995. A reexamination of the pollen-donation hypothesis in an experimental population of Asclepias exaltata. Evolution, 49, 89-99.

CROW, J. F. 1958. Some possibilities for measuring selection intensities in man. Hum. Biol, , 30, 1-13.

FALCONER, D. S. 1981. Introduction to Quantitative Genetics, 2nd edn. Longman, London.

GALIL, J. AND ZERONI, M. 1969. On the organization of the pollinium in Asclepias curassavica. Bot. Gaz., 130, 1-4.

KAHN, A. P. AND MORSE, D. H. 1991. Pollinium germination and putative ovule penetration in self- and cross-pollinated common milkweed Asclepias syriaca. Am. Midl. Nat., 126, 61-67.

LANDE, R. 1976. Natural selection and random genetic drift in phenotypic evolution. Evolution, 30, 314-334.

LANDE, R. 1979. Quantitative genetic analyses of multivariate evolution, applied to brain:body size allometry. Evolution, 33, 402-416.

LANDE, R. AND ARNOLD, s. J. 1983. The measurement of selection on correlated characters. Evolution, 36, $1210-1226$.

LLOYD, D. G. 1985. Parallels between sexual strategies and other allocation strategies. Experientia, 41, 1277-1285.

MORGAN, M. T. 1992. The evolution of traits influencing male and female fertility in outcrossing plants. Am. Nat., 139, 1022-1051.

MORGAN, M. T. 1994. Models of sexual selection in hermaphrodites, especially plants. Am. Nat., 144 (Suppl.), S100-S125.

MORSE, D. H. 1994. The role of self-pollen in the female reproductive success of common milkweed (Asclepias syriaca, Asclepiadaceae). Am. J. Bot., 81, 322-330.
MORSE, D. H. AND FRITZ, R. S. 1983. Contributions of diurnal and nocturnal insects to the pollination of common milkweed (Asclepias syriaca L.) in a pollenlimited system. Oecologia, 60, 190-197.

NILSSON, L. A., RABAKOHANDRIANINA, E. AND PETTERSSON, B. 1992. Exact tracking of pollen transfer and mating in plants. Nature, 360, 666-668.

PIPER, J. G. AND WAITE, s. 1988. The gender role of flowers of broad leaved Helleborine, Epipactis helleborine (L.) Crantz (Orchidaceae). Funct. Ecol., 2, 35-40.

PleAsanTs, J. M. 1991. Evidence for short-distance dispersal of pollinia in Asclepias syriaca L. Funct. Ecol., 5, $75-82$.

QUELLER, D. C. 1983. Sexual selection in a hermaphroditic plant. Nature, 305, 706-707.

QUELLER, D. C. 1985. Proximate and ultimate causes of low fruit production in Asclepias exaltata. Oikos, 44, 373-381.

ROBERTSON, C. 1886 . Notes of the mode of pollination of Asclepias. Bot. Gaz., 11, 262-269.

SHORE, J. S. 1993. Pollination genetics of the common milkweed, Asclepias syriaca L. Heredity, 70, 101-108.

SNOW, A. A. 1989. Assessing the gender role of hermaphroditic flowers. Funct. Ecol., 3, 249-250.

WEBER, K. E. 1992. How small are the smallest selectable domains of form? Genetics, 130, 345-353.

WILLSON, M. F. AND BERTIN, R. I. 1979. Flower visitors, nectar production, and inflorescence size of Asclepias syriaca. Can. J. Bot., 57, 1380-1388.

WILLSON, M. F. AND PRICE, P. W. 1977. The evolution of inflorescence size in Asclepias (Asclepiadaceae). Evolution, 31, 495-511.

WILLSON, M. F. AND RATHCKE, B. J. 1974. Adaptive design of the floral display in Asclepias syriaca L. Am. Midl. Nat., 92, 47-57.

wood, C. E., JR. 1974. A Student's Atlas of Flowering Plants: Some Dicotyledons of Eastern North America. Harper \& Row, New York.

wyatt, R. 1976. Pollination and fruit set in Asclepias: a reappraisal. Am. J. Bot., 63, 845-851.

WYATT, R. 1978. Experimental evidence concerning the role of the corpusculum in Asclepias pollination. Syst. Bot., 3, 313-321.

WYATT, R. 1981. The reproductive biology of Asclepias tuberosa. II. Factors determining fruit-set. New Phytol., 88, 375-385.

WYATT, R. AND BROYLES, S. B. 1990. Reproductive biology of milkweeds (Asclepias): recent advances. In: Kawano, S. (ed.) Biological Approaches and Evolutionary Trends in Plants, pp. 255-272. Academic Press, Toronto.

WYATT, R., BROYLES, S. B. AND DERDA, G. S. 1992. Environmental influences on nectar production in milkweeds (Asclepias syriaca and A. exaltata). Am. J. Bot., 79, 636-642. 